\title{
Effect of Nanoparticles on Electron and Thermoelectric Transport
}

\author{
MONA ZEBARJADI, ${ }^{1,5}$ KEIVAN ESFARJANI, ${ }^{2}$ ALI SHAKOURI, ${ }^{1}$ \\ ZHIXI BIAN, ${ }^{1}$ JE-HYEONG BAHK,${ }^{3}$ GEHONG ZENG,${ }^{3}$ JOHN BOWERS, ${ }^{3}$ \\ HONG LU, ${ }^{4}$ JOSHUA ZIDE, ${ }^{4}$ and ART GOSSARD ${ }^{4}$ \\ 1.-Department of Electrical Engineering, University of California, Santa Cruz, CA 95064, USA. \\ 2.-Department of Physics, University of California, Santa Cruz, CA 95064, USA. 3.-Electrical \\ and Computer Engineering Department, University of California, Santa Barbara, CA 93106, USA. \\ 4.-Materials Department, University of California, Santa Barbara, CA 93106, USA. 5.-e-mail: \\ mona@soe.ucsc.edu
}

\begin{abstract}
Recent experimental results have shown that adding nanoparticles inside a bulk material can enhance the thermoelectric performance by reducing the thermal conductivity and increasing the Seebeck coefficient. In this paper we investigate electron scattering from nanoparticles using different models. We compare the results of the Born approximation to that of the partial-wave method for a single nanoparticle scattering. The partial-wave method is more accurate for particle sizes in the $1 \mathrm{~nm}$ to $5 \mathrm{~nm}$ range where the point scattering approximation is not valid. The two methods can have different predictions for the thermoelectric properties such as the electrical conductivity and the Seebeck coefficient. To include a random distribution of nanoparticles, we consider an effective medium for the electron scattering using the coherent potential approximation. We compare various theoretical results with the experimental data obtained with ErAs nanoparticles in an InGaAlAs matrix. Reasonably good agreement is found between the measured and theoretical electrical conductivity and Seebeck data in the $300 \mathrm{~K}$ to $850 \mathrm{~K}$ temperature range.
\end{abstract}

Key words: Nanoparticle scattering, thermoelectric, transport

\section{INTRODUCTION}

In recent years, along with advancement in materials synthesis, it has been possible to embed nanoparticles with controlled size in bulk materials. Such structures can be used for various applications such as thermoelectric power generators ${ }^{1}$ and solar cells. ${ }^{2}$ The advantage of incorporating nanoparticles inside thermoelectric materials is to reduce the lattice thermal conductivity ${ }^{3}$ and enhance the Seebeck coefficient due to electron filtering. ${ }^{4}$ Adding nanoparticles also reduces the electrical conductivity. Therefore the size and the material forming the nanoparticles should be chosen in such a way that they scatter phonons more effectively than electrons.

(Received July 8, 2008; accepted December 31, 2008; published online January 28, 2009)
Scattering of electrons by localized defect potentials, ${ }^{5}$ by space-charge regions, ${ }^{6}$ and by neutral impurities ${ }^{7}$ have been widely studied. Busch and Soukoulis 8 investigated the transport of a classical wave in a random medium composed of dielectric spheres. They obtained the mean free path of the transport and the energy transport velocity, which were in agreement with the experimental results. Sheng ${ }^{9}$ calculated the electronic transport in granular metal films. His theory is extendable to the case of metallic spherical nanoparticles inside a host matrix. Kim and Majumdar ${ }^{10}$ calculated the phonon scattering cross section of spherical nanoparticles within the Born approximation, and found an oscillatory behavior in the scattering cross section, presumably due to acoustic mismatch. Recently, Faleev and Leonard ${ }^{11}$ showed enhancement of the power factor of semiconductors with metallic nanoinclusions at 
high doping concentrations. They have used the partial-wave technique to calculate the scattering rates from nanoparticles; however, the results are based on Eq. 35 in their paper, which is not correct (see Eq. 6 of this paper). In contrast to their prediction, we find that there is a big difference between a well potential and a barrier potential.

In this paper, we consider finite-size nanoparticles inside a host semiconductor and investigate the effect of adding nanoparticles on the thermoelectric transport using three different methods. We use the Born approximation and the partial-wave technique to calculate the scattering cross section and transport properties in the dilute limit (low concentration of nanoparticles). For higher concentrations we use the coherent potential approximation (CPA), ${ }^{12,13}$ which is known as the best single-site approximation for a disordered system. The predictions of these models of thermoelectric transport are studied in this work.

\section{MODEL AND THEORY}

\section{Single Nanoparticle Scattering}

Here we develop the theory to calculate the scattering cross section of a single nanoparticle. The theory is applicable to the case of any arbitrary spherically symmetric potential.

\section{Born Approximation}

The Born approximation is based on the perturbation theory and therefore has the intrinsic assumption that the potential of the scatterer is weak. This approximation works well for energies which are several times the barrier height. For a square barrier/well potential, the Born approximation yields the following results for the differential scattering cross section $(\sigma(\theta))$, the total scattering cross section $(\sigma)$, and the momentum cross section $\left(\sigma_{\mathrm{m}}\right)$, respectively. ${ }^{14}$

$$
\begin{gathered}
\sigma(\theta)=\left(\frac{2 m^{*} V_{0} a^{3}}{\hbar^{2}}\right)^{2} g\left(2 k a \sin \frac{\theta}{2}\right) \\
g(x)=\frac{(\sin x-x \cos x)^{2}}{x^{6}}, \\
\sigma=2 \pi \int \sigma(\theta) \sin \theta \mathrm{d} \theta=\frac{32 \pi m^{* 2} V_{0}^{2} a^{6}}{\hbar^{4}} \gamma(2 k a) ; \\
\gamma(x)=\frac{1}{4 x^{2}}\left(1-\frac{1}{x^{2}}+\frac{\sin 2 x}{x^{3}}-\frac{\sin ^{2} x}{x^{4}}\right), \\
\sigma_{\mathrm{m}}=2 \pi \int \sigma(\theta)(1-\cos \theta) \sin \theta \mathrm{d} \theta \\
=\pi\left(\frac{2 m^{*} V_{0} a}{\hbar^{2} k^{2}}\right)^{2} \int_{0}^{2 k a} \frac{(\sin x-x \cos x)^{2}}{x^{3}} \mathrm{~d} x,
\end{gathered}
$$

where, $a$ is the radius of the nanoparticle, $V_{0}$ is the barrier/well height, $k$ is the wavevector of the incident electron, and $m^{*}$ is its effective mass in the host material. As can be seen from these formulas, the sign of the potential (barrier or well) does not matter, and the strength of the nanoparticle potential (and its size, in the high-energy limit) comes as a multiplicative factor.

\section{The Partial-Waves Method}

The partial-waves method is a way to calculate the scattering from a spherically symmetric potential exactly. The details of the method can be found in standard textbooks. ${ }^{14}$ The result for the total cross section is well known. We find for the momentum cross section the expression shown in Eq. 6, which is in contrast to the (incorrect) results of Faleev and Leonard.

$$
\sigma(\theta)=\frac{1}{k^{2}}\left|\sum_{l=0}^{\infty}(2 l+1) \mathrm{e}^{i \delta_{l}} \sin \delta_{l} P_{l}(\cos \theta)\right|^{2},
$$

$$
\sigma=\frac{4 \pi}{k^{2}}\left[\sum_{l=0}^{\infty}(2 l+1) \sin ^{2} \delta_{l}\right],
$$

$$
\begin{aligned}
\sigma_{\mathrm{m}}=\frac{4 \pi}{k^{2}}\left[\sum_{l=0}^{\infty}(2 l+1) \sin ^{2} \delta_{l}\right. \\
\left.\quad-\sum_{l=0}^{\infty} 2 l \cos \left(\delta_{l}-\delta_{l-1}\right) \sin \delta_{l} \sin \delta_{l-1}\right], \\
\tan \delta_{l}=\frac{k j_{l}^{\prime}(k a)-y_{l} j_{l}(k a)}{k n_{l}^{\prime}(k a)-y_{l} n_{l}(k a)} \quad y_{l}=\left.\frac{\phi^{\prime}}{\phi}\right|_{r=a},
\end{aligned}
$$

where $\delta_{l}$ is called the phase shift of the $l$ th partial wave ( $l$ being the angular momentum quantum number) and $\phi$ is the wavefunction of the electron inside the nanoparticle. For a known potential shape and a given energy, the solution of the Schrodinger equation can be found numerically (for example, by the Numerov method) inside the nanoparticle. Then $y_{l}$ is calculated by matching the slope of the wavefunction at the boundary between the nanoparticle and the host material. We use the shooting method to find the wavefunction within the cutoff radius of the nanoparticle. The solution is exact and can be applied to any arbitrary spherically symmetric potential of finite range.

\section{Scattering from a Random Medium}

In the dilute limit, when the nanoparticles are far apart, each nanoparticle can be treated independently; however, when the concentration of nanoparticles increases, this approximation ceases 
to be valid. We need to add the effect of the nanoparticles volume, meaning that the electron wave sees a random medium instead of individual scatterers. One approach to solve such a problem is to use an effective medium theory. Instead of a random potential due to nanoparticles, the electron wave sees an effective medium. At each energy $E$, the system behaves as if a coherent potential or a selfenergy $\Sigma(E)$ was added to the host Hamiltonian. We use the CPA following the work of Sheng, ${ }^{9}$ but apply it to a bulk matrix with a given concentration of spherical nanoparticles. Figure 1 shows the model schematically. We assume that each nanoparticle is embedded inside a host material and they are both inside an effective medium. A distribution function is defined for the nanoparticles radii. The host radius is set according to the concentration of nanoparticles and their average radius. The T-matrix of each nanoparticle species in three dimensions is related to the amplitude of the scattered wave at infinity:

$$
\begin{aligned}
\phi & \stackrel{r \gg R}{\longrightarrow} \exp (i \vec{k} \cdot \vec{r})+\frac{f(\theta)}{r} \exp (i k r), \\
f(\theta) & =-\frac{1}{4 \pi}\left\langle k^{\prime}\left|t^{+}\right| k\right\rangle,
\end{aligned}
$$

The CPA condition ${ }^{15}$ :

$$
\operatorname{Re}\left(\sum_{m} c_{m}\left\langle k_{\mathrm{e}}\left|t_{m}^{+}\right| k_{\mathrm{e}}\right\rangle\right)=0
$$

states that, on average, the scattering of the (host + nanoparticle) in the effective medium is zero. The index $m$ refers to each different radius of the nanoparticle, $c_{m}$ is the weight of each species $m$, $t_{m}^{+}$is the retarded T-matrix for each species inside the effective medium, and $k_{\mathrm{e}}$ is the wavevector of the electron inside the effective medium. The total scattering cross section can be obtained from the optical theorem.

$$
\sigma=-\frac{1}{k_{\mathrm{e}}} \operatorname{Im} \sum_{m} c_{m}\left\langle k_{\mathrm{e}}\left|t_{m}^{+}\right| k_{\mathrm{e}}\right\rangle
$$



Fig. 1. Schematic diagram of the coherent potential model. The nanoparticle is a sphere embedded inside the host material (the outer sphere) and they are both inside an effective medium.
In the dilute limit the CPA results tend to that of the partial-wave method.

\section{Thermoelectric Transport}

For modeling a realistic material, we need to include in the formalism other scattering mechanisms such as phonons and impurities. The distribution function relaxation time $\tau_{\mathrm{f}}$ appearing in the collision term of the Boltzmann's equation can be obtained by summing the inverse of relaxation times for each individual scattering mechanism. For elastic scatterings the distribution function relaxation time is the same as the momentum relaxation time. Nanoparticle scatterings are assumed to be elastic. The relaxation time $\tau$ is inversely proportional to the total cross section. Thermoelectric properties can be obtained by using the linear response theory and by integrating the differential conductivity over energy:

$$
S=\frac{1}{e T}\left(\frac{\int v^{2}(E-\mu) \tau_{\mathrm{f}}(E) \operatorname{Dos}(E) \frac{\partial f}{\partial E} \mathrm{~d} E}{\int v^{2} \tau_{\mathrm{f}}(E) \operatorname{Dos}(E) \frac{\partial f}{\partial E} \mathrm{~d} E}\right)
$$

$$
\frac{1}{\rho}=-\frac{2 e^{2}}{3 m^{*}} \int E \tau_{\mathrm{f}}(E) \operatorname{Dos}(E) \frac{\partial f}{\partial E} \mathrm{~d} E,
$$

where $S$ is the Seebeck coefficient, $\rho$ is the resistivity, $\mu$ is the chemical potential, $\tau_{\mathrm{f}}$ is the distribution function relaxation time, $\operatorname{Dos}(E)$ is the density of states at energy $E, T$ is the temperature, and $f(E)$ is the Fermi-Dirac distribution function.

\section{RESULTS AND DISCUSSION}

First, we look at the dilute limit, where the nanoparticles can be considered as independent potentials. As we mentioned before, the Born approximation is valid for weak potentials. Equations 1-3 show that the scattering cross section is proportional to $V_{0}^{2}$. This means that the barrier height is just a scaling factor and that the result of the Born approximation is not sensitive to the attractive or repulsive nature of the potential. The radius of the nanoparticle is another scaling factor and it does not change the behavior of the scattering cross section versus energy at high energies. In Fig. 2 we have compared the scattering cross sections resulting from the Born approximation and the partial-wave method.

In this case the Born approximation overestimates the scattering cross sections of a barrier potential and underestimates that of a well potential. Within the Born approximation, forward scattering is independent of the electron energy. However, the partial-wave method predicts that forward scattering increases with energy, which means that lowenergy electrons are more affected by the potential compared with high-energy ones. 

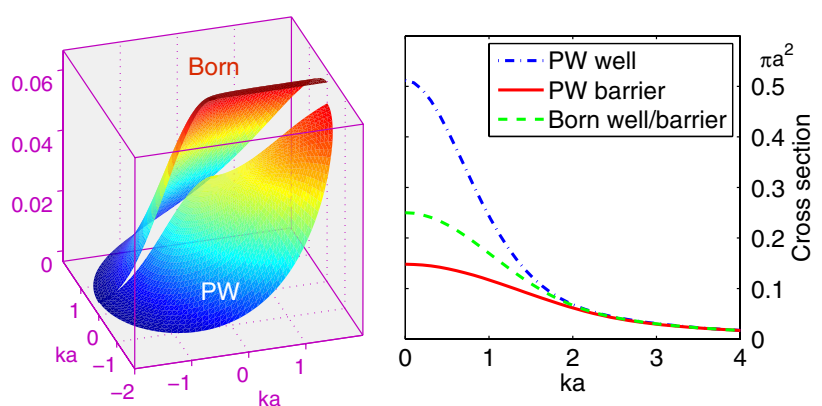

Fig. 2. Comparison of the Born approximation and the partial-wave method. Right: scattering cross section in units of $\pi a^{2}$ for the partialwave and the Born approaches. The barrier/well energy scale $\left(2 \mathrm{Va}^{2}\right)$ here is 0.75 . Left: differential scattering cross section as a function of electron momentum (the horizontal axis is in the direction of the incident wave and the vertical axis is in the transverse direction). We assumed a potential barrier. The differential scattering cross section is shown for the partial-wave method (top right) and for the Born approximation (bottom right).

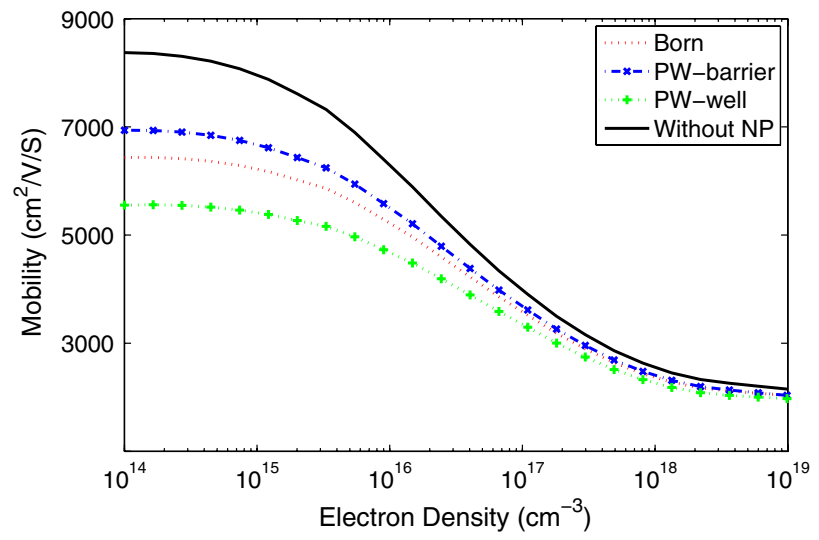

Fig. 3. Mobility versus electron concentration using the partialwaves method and the Born approximation. Nanoparticle potentials are considered as square barriers/wells of height $0.1 \mathrm{eV}$ and a radius of $2 \mathrm{~nm}$. Mobilities are shown for the case of $1 \%$ nanoparticle concentration. The host material is GaAs for which the results without nanoparticles are also reported for comparison and also to validate our model in comparison with experiments.

Figure 3 shows the mobility prediction obtained using the two approaches. This figure is plotted for the case of GaAs. In this calculation we consider other important scattering mechanisms such as polar optical phonons, acoustic phonons, and ionized impurities in addition to the nanoparticle scattering. The results without nanoparticles are also reported. They are in good agreement with the experimental results. ${ }^{16}$ Notice that the Born underestimates/ overestimates the mobility by about $7 \% / 15 \%$ compared with that of the partial-wave method for the case of barrier/well potential. The partial-wave method is accurate for low concentrations when multiple scatterings can be ignored. For higher concentrations we need to go beyond the single nanoparticle picture. The electron wave sees an effective medium instead of a single independent scatterer. We take into account the fluctuations of the random medium by considering a distribution func-

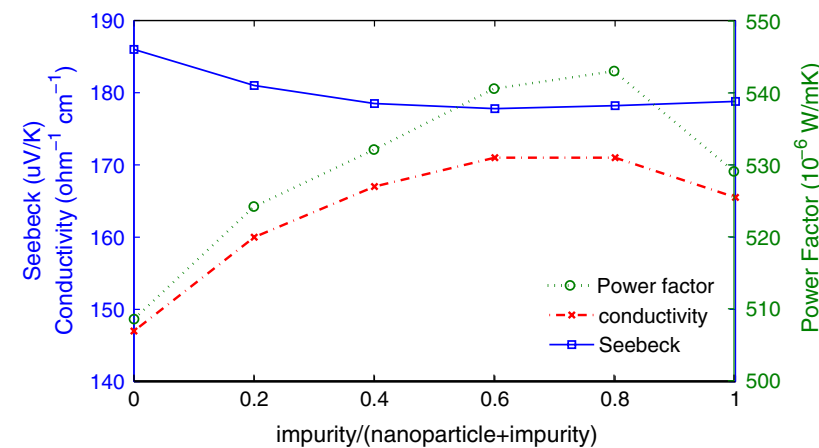

Fig. 4. CPA results for InGaAIAs with $1 \% \mathrm{Er}$ at room temperature. A Gaussian distribution was assumed for nanoparticles with an average radius of $1.5 \mathrm{~nm}$ and deviation of $0.5 \mathrm{~nm}$. The carrier concentration is $7 \times 10^{17} \mathrm{~cm}^{-3}$

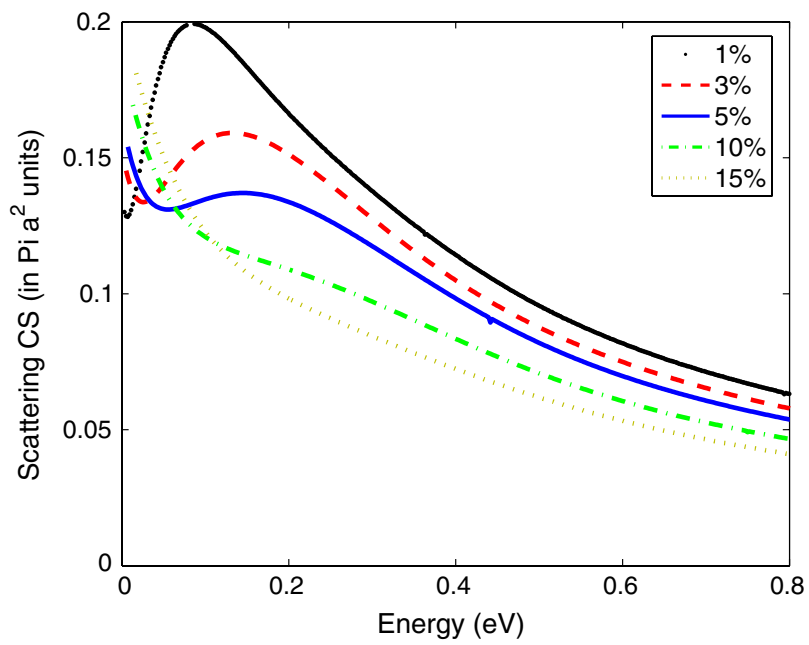

Fig. 5. Total scattering cross section per nanoparticle for different nanoparticle concentrations using the CPA. The nanoparticle radius distribution function is a Gaussian function with an average radius of $2 \mathrm{~nm}$ and variance of $0.7 \mathrm{~nm}$. The barrier height is $0.1 \mathrm{eV}$.

tion for nanoparticle radii and averaging over it (see also Eqs. 9 and 10). Figure 4 shows the CPA prediction for the thermoelectric properties for a sample material. We did not optimize the power factor but generally we observed that the enhancement of the Seebeck coefficient is higher for higher carrier concentrations but there is an optimum concentration for the highest thermoelectric power factor.

To simplify the discussion let us take the square barrier/well model again. To stay close to the parameters of previous methods (Fig. 3), we choose the same material ( $\mathrm{GaAs}$ ) with the same barrier height for nanoparticles $(0.1 \mathrm{eV})$. The nanoparticle radius distribution function is chosen such that the average radius of the nanoparticles stays the same as before $(2 \mathrm{~nm})$. Figure 5 shows the total scattering cross section per nanoparticle for different nanoparticle concentrations. Similar to the other two methods, the scattering cross section goes to zero as $E^{-1}$ for large $E$. It can be seen from the figure that, for low concentrations, electrons with energies close to the barrier height $(0.1 \mathrm{eV})$ have the 
largest cross section. At high nanoparticle concentrations, however, electrons see an overall potential distribution and do not see single barriers. Therefore the peak at $0.1 \mathrm{eV}$ disappears in favor of a sharp increase at low energies. Such a sharp increase is an indication of the electron filtering. Lowenergy electrons are blocked in this picture and high-energy ones contribute to the transport, and therefore the average energy transported per carrier (thermopower) is increased. The CPA method results in imaginary wavenumbers inside the effective medium below a threshold energy. This means that these states are localized and do not contribute to the transport. We can then define a mobility edge, above which states are extended and below which states are localized. We have observed that at low concentrations this edge is close to $c V_{0}$ (the concentration of nanoparticles multiplied by the barrier height is the mean band edge in the medium), whereas at higher concentrations the edge has slightly higher values.

Figure 6 shows the mobility versus electron density for different nanoparticle concentrations. In
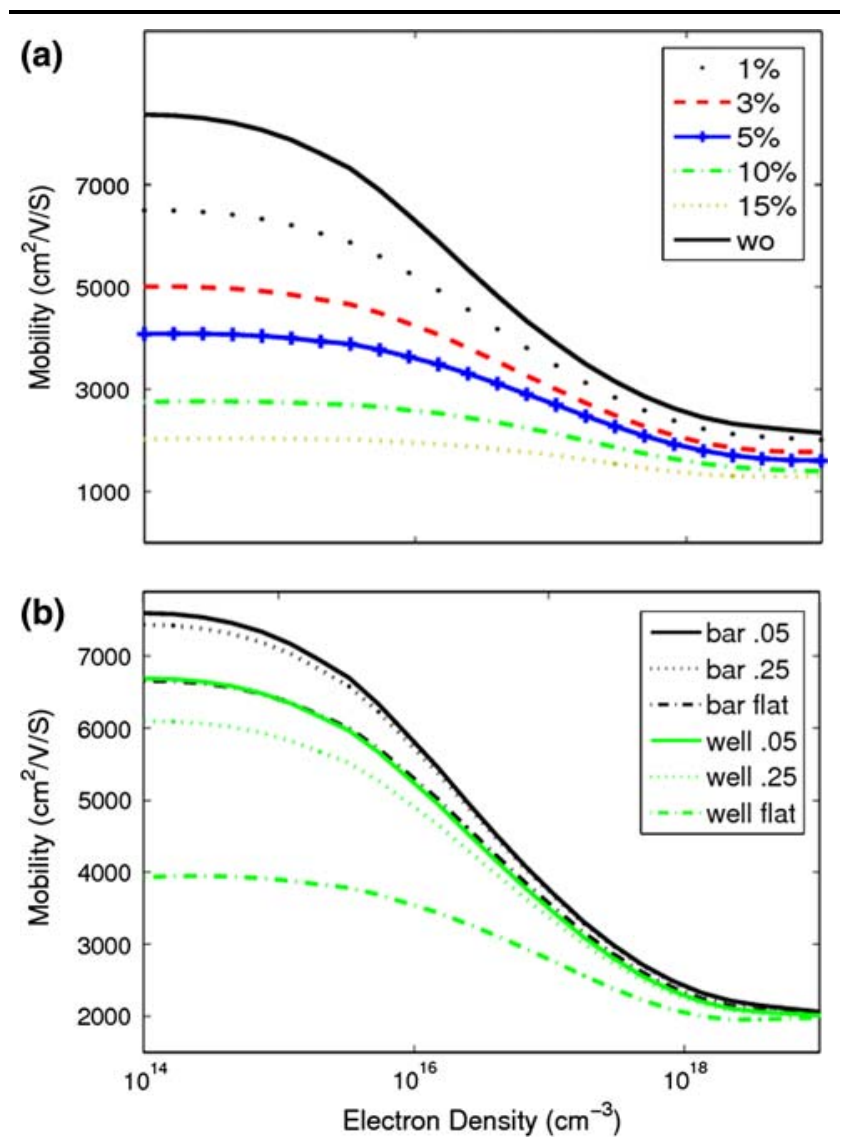

Fig. 6. Mobility versus carrier concentration using the CPA method. Top: for different nanoparticle concentrations. Mobility without nanoparticles ("wo") is also plotted. Parameters are the same as Fig. 5. Bottom: for different size distributions. Solid and dotted lines are for Gaussian functions with variance of $0.05 \mathrm{~nm}$ and $0.25 \mathrm{~nm}$, respectively. Dotted dashed lines are for a flat distribution if radii between $1 \mathrm{~nm}$ and $3 \mathrm{~nm}$. Results are reported for both barrier and well potentials. Nanoparticle concentration is $1 \%$. these calculations, the electron density is increased by increasing the concentration of ionized impurities. If the randomness of the system is increased, for example by changing the nanoparticle radius distribution function from a Gaussian to a flat distribution, the nanoparticle scattering rate increases as expected and therefore the mobility decreases. Figure $6 \mathrm{~b}$ shows that such changes affect the scattering form well potentials more than that of the barrier ones. This figure is plotted for $1 \%$ concentration of nanoparticles. If the concentration decreases to $0.3 \%$ or less, results of the CPA tend to that of the partial-wave approach.

\section{COMPARISON BETWEEN THEORY AND EXPERIMENT}

To verify the effect of nanoparticles on thermoelectric transport we made two samples. The samples were InGaAlAs with $20 \%$ InAlAs grown on an InP substrate. The substrate was then removed and the samples were bonded to sapphire plates. One sample was bulk InGaAs doped with $2 \times 10^{18} \mathrm{~cm}^{-3}$ concentration of silicon. To the other sample $0.3 \%$ Er nanoparticles were added. ErAs nanoparticles are formed inside the bulk matrix with sizes of $2 \mathrm{~nm}$ to $3 \mathrm{~nm}$. Hall measurement was done to obtain the carrier concentration of the samples. In-plane Seebeck and conductivity were measured and are reported in Fig. 7.

To fit the data with our theory, we set the material properties of InGaAlAs by interpolating between InGaAs and InAlAs. The conductivity data of bulk InGaAlAs was used to set the scattering rates by fitting the rates to a power series in electron energy. Detailed inclusion of all the scattering mechanisms for bulk InGaAlAs will be presented elsewhere. Nanoparticle scattering rates are then added to these rates. Nanoparticles are assumed to have a repulsive screened potential away from the interface $\frac{z}{r r} \mathrm{e}^{-\lambda r} . \varepsilon$ is the dielectric constant, $1 / \lambda$ is the screening length, and $z$ is the number of electrons per nanoparticle contributing to the conduction band. Inside the nanoparticle a uniform distribution of $z+$ charges was assumed. A part of the erbium does not form nanoparticles; rather it forms pointlike potentials (ionized dopants). The ratio of carriers coming from dopants to those coming from nanoparticles was used as a fitting parameter. Total number of carrier concentration was set from the Hall data. Figure 7 shows the comparison between the prediction of the theory and the experimental data. The model is able to reproduce conductivity data quite accurately. The Seebeck coefficient can be off by as much as $15 \%$. One can note a systematic underestimation, however, in the case of nanoparticle doping. The difference may come from the fact that the real potential of the nanoparticles is not known and that we have used a simplified model of the potential distribution around nanoparticles. 

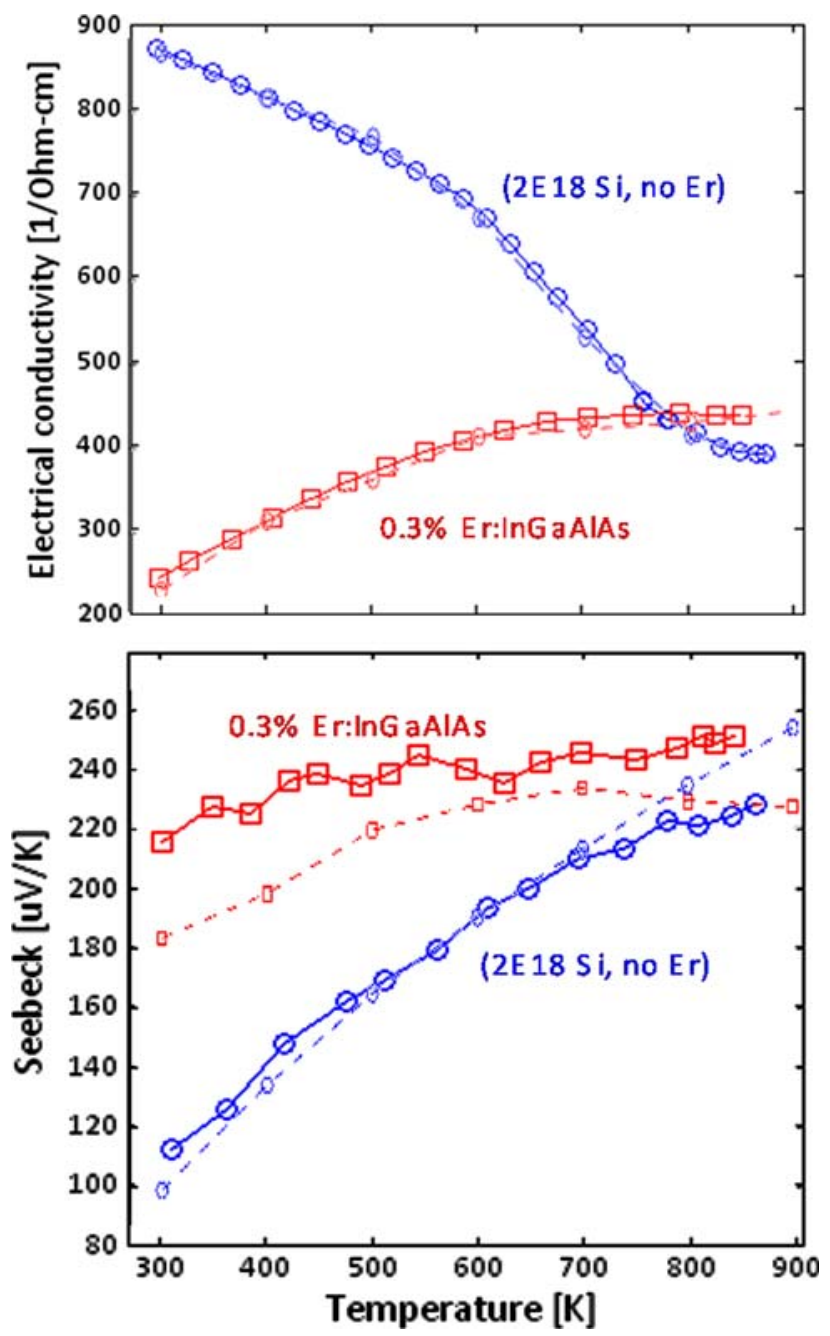

Fig. 7. Comparison of the theory with the experiment: Solid lines are experimental data and dashed lines are theoretical prediction. The model is explained in the text. Electrical conductivities were fitted (using the appropriate ratio of dopant carriers to nanoparticle carriers), and the Seebeck coefficient was predicted.

\section{CONCLUSIONS}

The Born approximation cannot quantitatively predict transport properties of a bulk matrix with embedded nanoparticles; it is not sensitive to the attractive or repulsive nature of the potential. The prediction of the Born approximation is not correct for low energies. Using the partial-wave method, scattering at low energies is isotropic but the Born approximation predicts a dominant forward scattering for the whole energy range. In the dilute limit, the partial-wave method is a good approximation. For higher concentrations, CPA is more accurate. The results of the CPA tend to that of the partial-wave method in the dilute limit. CPA includes several classes of multiple scatterings off of different nanoparticles, but neglects processes involving more than four successive scatterings off of two different nanoparticles. This theory can be used to predict thermopower at higher nanoparticle concentrations. Nanoparticles can increase or decrease the power factor. This depends on their potential profile inside the bulk matrix and also on the position of the Fermi level. Preliminary experimental results show that the existing theory can explain the measured Seebeck coefficient and electrical conductivity of InGaAlAs samples with $0.3 \%$ volume concentration of ErAs nanoparticles with an average diameter of $1 \mathrm{~nm}$ to $2 \mathrm{~nm}$.

\section{ACKNOWLEDGEMENTS}

We are grateful to Dr. N. Mingo for discussions. This study was supported by ONR MURI Thermionic energy conversion center.

\section{OPEN ACCESS}

This article is distributed under the terms of the Creative Commons Attribution Noncommercial License which permits any noncommercial use, distribution, and reproduction in any medium, provided the original author(s) and source are credited.

\section{REFERENCES}

1. G.H. Zeng, J. Bahk, J.E. Bowers, J.M.O. Zide, A.C. Gossard, Z.X. Bian, R. Singh, A. Shakouri, W. Kim, S.L. Singer, and A. Majumdar, Appl. Phys. Lett. 91, 263510 (2007). doi: 10.1063/1.2828042.

2. B. Tan and Y. Wu, J. Phys. Chem. B 110, 15932 (2006). doi:10.1021/jp063972n.

3. W. Kim, S.L. Singer, A. Majumdar, D. Vashaee, Z.X. Bian, A. Shakouri, G.H. Zeng, J.E. Bowers, J.M.O. Zide, and A.C. Gossard, Appl. Phys. Lett. 86, 242107 (2006). doi: 10.1063/1.2207829.

4. G.H. Zeng, J.M.O. Zide, W. Kim, J.E. Bowers, A.C. Gossard, Z.X. Bian, Y. Zhang, A. Shakouri, S.L. Singer, and A. Majumdar, J. Appl. Phys. 101, 034502 (2007). doi: 10.1063/1.2433751.

5. B. Podor, Phys. Rev. B 26, 2551 (1983). doi:10.1103/Phys RevB.27.2551.

6. L.R. Weisberg, J. Appl. Phys. 33, 1817 (1962). doi:10.1063/ 1.1728839 .

7. N. Sclar, Phys. Rev. 104, 1559 (1956). doi:10.1103/Phys Rev.104.1559.

8. K. Busch and C.M. Soukoulis, Phys. Rev. Lett. 75, 3442 (1995). doi:10.1103/PhysRevLett.75.3442.

9. P. Sheng, Philoso. Mag. B 65, 357 (1992). doi:10.1080/ 13642819208207638.

10. W. Kim and A. Majumdar, J. Appl. Phys. 99, 084306 (2006). doi:10.1063/1.2188251.

11. S.V. Faleev and F. Leonard, Phys. Rev. B 77, 214304 (2008). doi:10.1103/PhysRevB.77.214304.

12. P. Soven, Phys. Rev. 156, 809 (1967). doi:10.1103/PhysRev.156.809.

13. D.W. Taylor, Phys. Rev. 156, 1017 (1967).

14. L.I. Schiff, Quantum Mechanics (New York: McGraw-Hill, 1949).

15. P. Sheng, Introduction to Wave Scattering, Localization, and Mesoscopic Phenomena (New York: Academic, 1995).

16. D.L. Rode, Semiconductors and Semimetals, Vol. 10, ed. R.K. Willardson and A.C. Beer (New York: Academic, 1975). 